\title{
Introduction to the Business Rule Management Technologies Minitrack
}

\author{
Carl Corea \\ University of Koblenz-Landau \\ ccorea@uni-koblenz.de
}

\author{
Faruk Hasić \\ KU Leuven \\ faruk.hasic@kuleuven.be
}

\author{
Patrick Delfmann \\ University of Koblenz-Landau \\ delfmann@uni-koblenz.de estefania.serral@kuleuven.be
}

\begin{abstract}
Business rules are currently evolving into a central artifact for enabling Business Process Management and Compliance Management. While standards for rule modeling such as Decision Model and Notation (DMN) are more and more maturing, the actual management of business rules seems strongly neglected in research. This track therefore welcomes submissions that address business rules management (BRM) in organizations
\end{abstract}

\section{Introduction}

In this edition of HICSS, we are happy to introduce the novel minitrack 'Business Rule Management Technologies' at HICSS. Business rules (BRs) in general have long been acknowledged as an important research discipline in relation with business process management and compliance management. In this track, we focus on the actual creation and long-term maintenance of business rules in organizational contexts, i.e., business rule management (BRM).

To clarify, consider the following exemplary BRM lifecycle in Figure 1.

\begin{tabular}{|c||c|c|}
\hline Align & \multicolumn{2}{|c|}{ Creation \& Maintenance } \\
\hline Plan & Capture & Implementation \\
\hline
\end{tabular}

Figure 1. Exemplary BRM lifecycle, taken from [1]

While there have been various concrete BRM lifecycle models proposed, they can typically be divided into the phases as shown: (1 - align) BRM comprises strategic alignment of $\mathrm{BRs}$ within the organisation. Then (2 - creation/maintenance) BRM comprises the actual creation and maintenance of BRs, e.g., capturing business rules (from legal texts or the like), authoring/formalizing business rules in a machine accessible form, and organizing business rules, i.e., ensuring error-freeness within the set of business rules, mostly by means of verification and re-modelling. Finally, (3 - implementation) BRM comprises the actual integration, application and monitoring of BRs. In all of these phases, there has been a wealth of recent works, e.g., [2, 3] (strategic alignment), [4, 5, 6, 7, 8] (capturing/mining), [9, 10, 11, 12] (verification), [13, 14] (monitoring and analysis), to name a few. As it seems, the topicality of business rules management seems to have gained recent interest in the community. Therefore, the motivation of this track is to provide a platform for BRM research. We invite works that address this challenge, aimed to support companies in business rule management. We welcome works from a variety of theoretical backgrounds such as qualitative research, design science research or case-studies. Research on state-of-the art rule management systems and tools is also encouraged. The topics of interest are in particular, but not limited to:

- Strategic alignment of BRM, e.g., maturity models, organizational or economic aspects regarding the implementation of BRM

- Rule capturing and mining

- (Collaborative) rule modelling

- Rule organizing and verification

- Rule integration into (collaborative) processes

- Rule-based system automation

- Rule-based model query and compliance monitoring

- Business rule management lifecycle approaches

\section{Presented Papers}

We are very happy that this minitrack seems to have been well-received, with a high number of submissions, despite the ongoing COVID-19 situation and this year being the first year of this minitrack. In accordance with 
an international program committee, we could accept 3 papers, which we outline in the following.

- Sam Leewis, Koen Smit, Matthijs Berkhout, BRM and Decision Mining - Filling in the Gaps. In this paper, the authors study the integration of BRM and DM through a simulation using a government and a healthcare case. During this simulation, three entry points are presented that describe how decision-related data should be utilized between BRM capabilities and DM phases to be able to integrate them.

- Maximilian Michel, Djordje Djurica, Jan Mendling, Identification of Decision Rules from Legislative Documents Using Machine Learning and Natural Language Processing. In this paper, the authors present an approach for using machine learning and natural language processing approaches to identify decision rules within legislative documents.

- Simon Vandevelde, Benjamin Callewaert, Joost Vennekens, Context-Aware Verification of DMN. In this paper, in addition to presenting a context-aware verification tool, the authors present two forms of context in decision models, namely in-model context and background knowledge.

We again congratulate the authors. Unfortunately, HICSS must be held digitally this year due to the ongoing situation. Nevertheless, the papers will be presented in a synchronous web-meeting during the conference time frame.

\section{Outlook and Acknowledgements}

Due to the high number of submissions, we are confident that we can offer this minitrack again for HICSS 2023. We thank the track chairs of the 'Organizational Systems and Technologies' track for including us in this year's program. We would also like to thank all the reviewers for their high efforts and contributions to the quality of this minitrack.

\section{References}

[1] C. Corea and P. Delfmann, "A taxonomy of business rule organizing approaches in regard to business process compliance," Enterprise Modelling and Information Systems Architectures (EMISAJ), vol. 15, pp. 4-21, 2020.

[2] R. Post, K. Smit, and M. Zoet, "Adoption and implementation of the decision model and notation standard," Journal of Advanced Management Science Vol, vol. 8, no. 2, 2020.
[3] S. Schlosser, E. Baghi, B. Otto, and H. Oesterle, "Toward a functional reference model for business rules management," in 2014 47th Hawaii International Conference on System Sciences, pp. 3837-3846, IEEE, 2014.

[4] V. Etikala and J. Vanthienen, "An overview of methods for acquiring and generating decision models," in International Conference on Knowledge Science, Engineering and Management, pp. 200-208, Springer, 2021.

[5] A. Alman, C. Di Ciccio, D. Haas, F. M. Maggi, and A. Nolte, "Rule mining with rum," in 2020 2nd International Conference on Process Mining (ICPM), pp. 121-128, IEEE, 2020.

[6] B. Scheibel and S. Rinderle-Ma, "Comparing decision mining approaches with regard to the meaningfulness of their results," arXiv preprint arXiv:2109.07335, 2021.

[7] J. De Smedt, F. Hasić, S. K. L. M. vanden Broucke, and J. Vanthienen, "Holistic discovery of decision models from process execution data," Knowledge-Based Systems, vol. 183, pp. 1-15, 2019.

[8] F. Hasić, J. De Smedt, S. Vanden Broucke, and E. Serral, "Decision as a Service (DaaS): A Service-Oriented Architecture Approach for Decisions in Processes," IEEE Transactions on Services Computing, pp. 1-14, 2020.

[9] C.-C. Grohé, C. Corea, and P. Delfmann, "Dmn 1.0 verification capabilities: An analysis of current tool support," in International Conference on Business Process Management, pp. 37-53, Springer, 2021.

[10] D. Calvanese, M. Dumas, Ü. Laurson, F. Maggi, M. Montali, and I. Teinemaa, "Semantics, analysis and simplification of DMN decision tables," Information Systems, vol. 78, pp. 112-125, 2018.

[11] F. Hasić, C. Corea, J. Blatt, P. Delfmann, and E. Serral, "Decision model change patterns for dynamic system evolution," Knowledge and Information Systems, vol. 62, no. 9, pp. 3665-3696, 2020.

[12] F. Hasić, C. Corea, J. Blatt, P. Delfmann, and E. Serral, "A tool for the verification of decision model and notation (DMN) models," in Research Challenges in Information Science (RCIS) 2020, vol. 385 of LNBIP, pp. 536-542, Springer, 2020.

[13] M. Gall and S. Rinderle-Ma, "Evaluating compliance state visualizations for multiple process models and instances," in International Conference on Business Process Management, pp. 126-142, Springer, 2021.

[14] G. De Giacomo, R. De Masellis, F. M. Maggi, and M. Montali, "Monitoring constraints and metaconstraints with temporal logics on finite traces," arXiv preprint arXiv:2004.01859, 2020. 